Int. J. Dev. Biol. 61: 235-244 (2017)

doi: $10.1387 / \mathrm{ijdb} .160235 \mathrm{ak}$

\title{
Roles of DSCAM in axonal decussation and fasciculation of chick spinal interneurons
}

\author{
OKSANA COHEN ${ }^{1}$, LILACH VALD ${ }^{1}$, MASAHITO YAMAGATA², JOSHUA R. SANES ${ }^{2}$ and AVIHU KLAR ${ }^{*, 1}$ \\ ${ }^{1}$ Dept. of Medical Neurobiology, IMRIC, Hebrew University-Hadassah Medical School, Jerusalem, Israel and \\ ${ }^{2}$ Center for Brain Science, Harvard University, Cambridge, Massachusetts, USA
}

\begin{abstract}
The ventral midline of the embryonic neural tube, the floor plate, has a profound role in guiding axons during embryonic development. Floor plate-derived guidance cues attract or repel axons, depending on the neuronal subtype and developmental stage. Netrin-1 and its receptor, Deleted in Colon Carcinoma (DCC), are the key constituents of commissurral axons guidance cues toward the floor plate. Recent studies have implicated Down Syndrome Cell Adhesion Molecule (Dscam) as an additional Netrin-1 receptor. In this study, we examined the role of Dscam in guiding defined spinal dorsal interneuron populations. In vivo knockdown and ectopic expression of Dscam were performed in the dorsal dl1, dl2 and dl3 interneurons of chick embryos, by separately increasing or decreasing Dscam expression in each of these three specific interneuronal populations. Neuron-specific gain and loss of function of Dscam had no effect on the axonal trajectories of dl1-3 neurons. The commissural neurons, dl1c and dl2, crossed the midline, and the ipsilaterally projecting neurons, dl1 $\mathrm{i}$ and dl3, projected ipsilaterally. However, the fasciculation of dl1 axons was diminished when Dscam expression was attenuated. Dscam is not required for either attraction to or repulsion from the floor plate. In contrast, Dscam is required for the fasciculation of axons, probably via homophilic interaction.
\end{abstract}

KEY WORDS: spinal cord, axon guidance, floor plate, DSCAM, fasciculation

\section{Introduction}

In bilaterally symmetric vertebrates, the floor plate at the ventral midline plays a crucial role in directing axon growth during the formation of neuronal circuits within the central nervous system. Floor plate cells provide a variety of guidance cues for growing axons, that elicit divergent effects depending on the neuronal cell type or its developmental stage (Dickson and Zou, 2010, Evans and Bashaw, 2010). Biochemical, in vitroand genetic evidence has demonstrated that the floor plate protein, Netrin-1, and its axonal receptor, Fra/DCC, are a key ligand-receptor pair that guides axons toward the floor plate (Dickson and Zou, 2010; Evans and Bashaw, 2010; Kennedy et al., 1994; Serafini et al., 1996; Serafini et al., 1994). In vertebrates, Shh and VEGF, which are expressed in the floor plate, and their axonal receptors, Boc, Ptc and Flk1, are additional attractive cues that synergize with netrin-1 in directing commissural growth cones toward the floor plate (Charron et al., 2003, Okada et al., 2006, Ruiz de Almodovar et al., 2011).
The incomplete failure of commissural midline crossing in $D C C$ mutant mice suggests that other receptors may convey the Netrin-1 signal (Fazeli et al., 1997). Several biochemical and genetic results implicated the Down Syndrome Cell Adhesion Molecule (Dscam) as a receptor for Netrin-1 that participates in regulation of midline crossing: 1) Netrin-1 binds to Dscam and DCC at similar affinities (Andrews etal., 2008, Liu etal., 2009, Ly etal., 2008). 2) Knockdown of Dscam by siRNA in mouse and chick embryonic neural tubes causes a substantial reduction in the number of commissural axons that cross the midline (Liu et al., 2009, Ly et al., 2008). 3) In an in vitro turning assay using rat neural tube explants, blocking either DCC or Dscam activity does not alter the turning of commissural axons within the neuroepithelium toward an exogenous Netrin-1 source, whereas blockade of both receptors prevents turning (Ly et al., 2008). 4) In a similar assay using chick neural tube explants,

Abbreviations used in this paper: DCG, deleted in colon carcinoma; Dscam, Down Syndrome cell adhesion molecule.

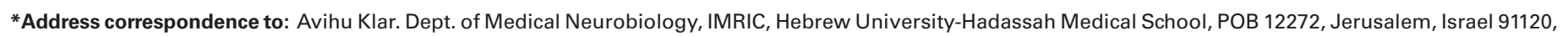

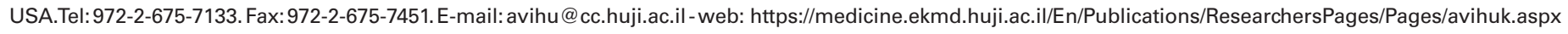


inhibition of Dscam by expression of siRNA or a dominant negative form of Dscam is sufficient to block the turning (Liu et al., 2009). 5) Expression of Dscam in Xenopus neurons mediates turning toward a Netrin-1 gradient in the absence of DCC signaling (Ly et al., 2008). 6) In flies, Netrin mutants have a similar phenotype to Dscam mutants. Additionally, overexpression of Dscam in axons that do not normally cross the midline is able to induce ectopic midline crossing (Andrews et al., 2008).

All these data are consistent with Dscam as an attractive receptor for Netrin-1. However, genetic analysis of Dscam mutants in mice has provided only partial support for this idea. In one Dscam allele, $D s c a m^{2 J}$, increased number of spinal commissural interneurons was apparent, and mutant mice displayed motor hypertonia and lack of locomotor coordination (Lemieux et al., 2016, Thiry et al., 2016). In another allele, commissural axons reach and cross the floor plate and in vitro they show normal outgrowth in response to Netrin-1 (Palmesino et al., 2012). One difference is that the $D s c a m^{2 J}$ allele may not be a null: it contains a four base pair duplication in exon 19, leading to a frameshift and truncation of the open reading frame. Hence, it is not clear whether the $D s \mathrm{sam}^{2 J}$ is

a hypomoroph or a neomorph.

Another possible explanation for the discrepancies is that specific subpopulations of spinal interneurons might differ in their dependence on Dscam, with alterations in one group masked by dispensability of Dscam for other groups. To explore this possibility, we selectively marked and manipulated three separate populations of dorsal spinal interneurons in chick: $\mathrm{dl} 1$, dl2 and dl3. The recent use of neuron-specific enhancer elements, which enables tracking of defined interneuron populations, has revealed that the dorsal interneurons dl1-3 have a different laterality preference (Avraham et al., 2010a, Avraham et al., 2009, Gowan et al., 2001, Helms and Johnson, 1998, Reeber et al., 2008). dl1 neurons project axons both ipsilaterally and contralaterally (Avraham et al., 2009, Wilson et al., 2008), dl2 have mostly commissural axons, while dl3 project their axons ipsilaterally (Avraham et al., 2010a, Avraham et al., 2009). In each case, we performed both loss- and gain-offunction tests, by either knock-down or elevation of Dscam levels in an interneuron-specific manner. We found that dl1-, dl2- and dl3-specific manipulations of Dscam expression did not change the trajectory choice of each of the dorsal interneuron populations. However, Dscam was required for proper axon fasciculation.

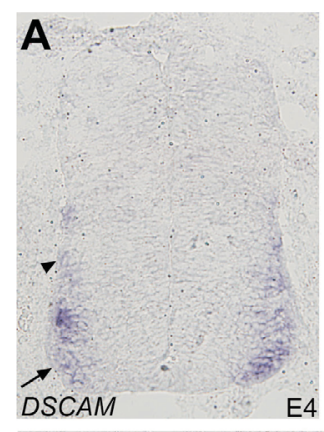

C
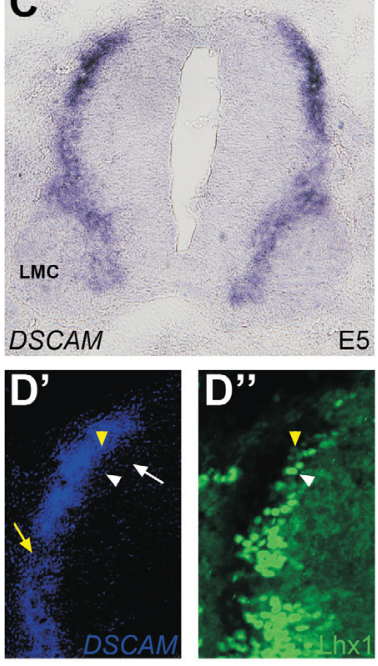

E

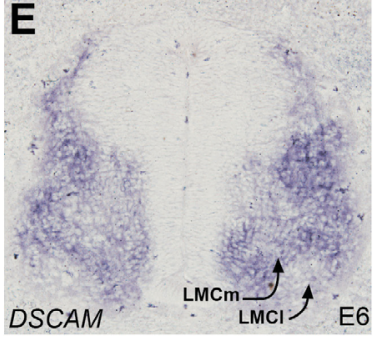

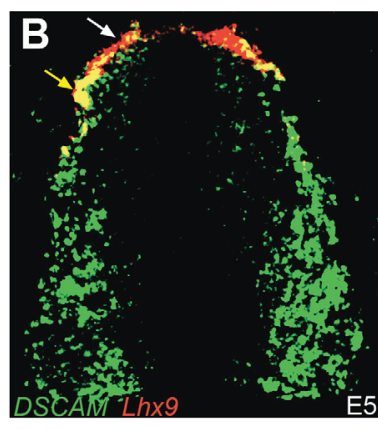
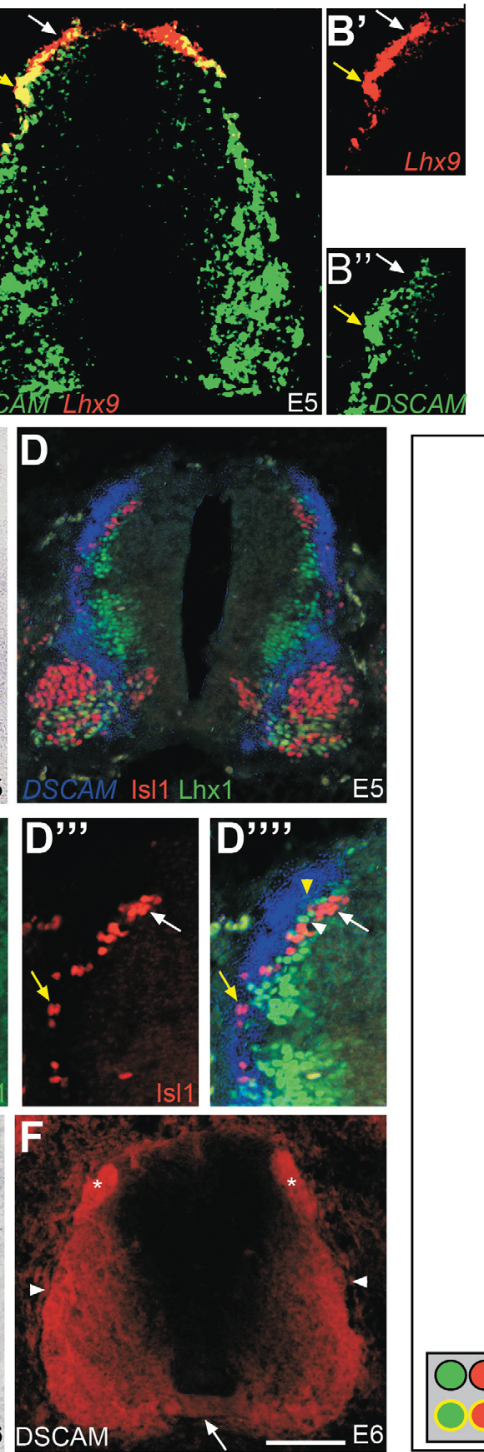

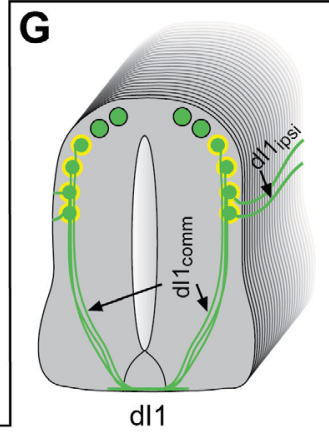

dl1
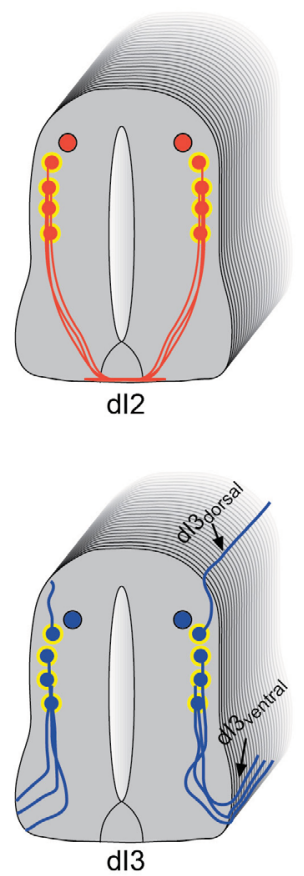

Early differentiated IN IN expressing DSCAM

\section{Results}

\section{Expression pattern of Dscam in the embryonic chick spinal cord}

To explore whether Dscam contributes to axon growth and guidance in the chick

Fig. 1. Expression pattern of Down Syndrome cell adhesion molecule (Dscam). In situ hybridization (A-E) and immunohistochemistry (F) were performed on cross sections of chick embryos. (A) At E4 Dscam is expressed in motor neurons (arrow) and in ventral interneurons (arrowhead). (B) Double in situ hybridization with Dscam (green) and Lhx9 (red). Dscam is not expressed in the early-differentiated dI1 neurons (white arrow), but is expressed in the differentiated dl1 neurons (yellow arrow). (C) In situ hybridization in the lumbar level of an E5 embryo. (D) The section in (C) was stained with antibodies against Lhx1/5 and Is/1. The bright-field image of (C) was photo-converted to a dark-field image. The earlydifferentiated d/2 neurons (white arrowhead) are $L h \times 1 / 5+/$ Dscam-, while the differentiated dl2 neurons (yellow arrowhead) are Lhx1/5+l Dscam + (D', D"). The early-differentiated dl3 neurons (white arrow) are Is/1+/Dscam-, while the differentiated d/3 neurons (yellow arrow) are Is/1+/Dscam+ (D'"', D'"')). (E) At E6, Dscam is expressed at low levels in $L M C l$ neurons and high levels in $L M C m$ neurons. (F) Dscam protein is detected on axons that cross the floor plate (arrow), project ipsilaterally (arrowheads) and at the dorsal root entry zone (asterisks). (G) Scheme summarizing the projection pattern of dl1-3 neurons and the expression of Dscam in those neurons. Scale bar in (F) equivalent to 100 $\mu m$ for $(D), 60 \mu m$ for $\left(D^{\prime}-D^{\prime \prime \prime \prime \prime}\right), 200 \mu m$ for $(A)$, $125 \mu \mathrm{m}$ for (B) and $75 \mu \mathrm{m}$ for $(E, F)$. 

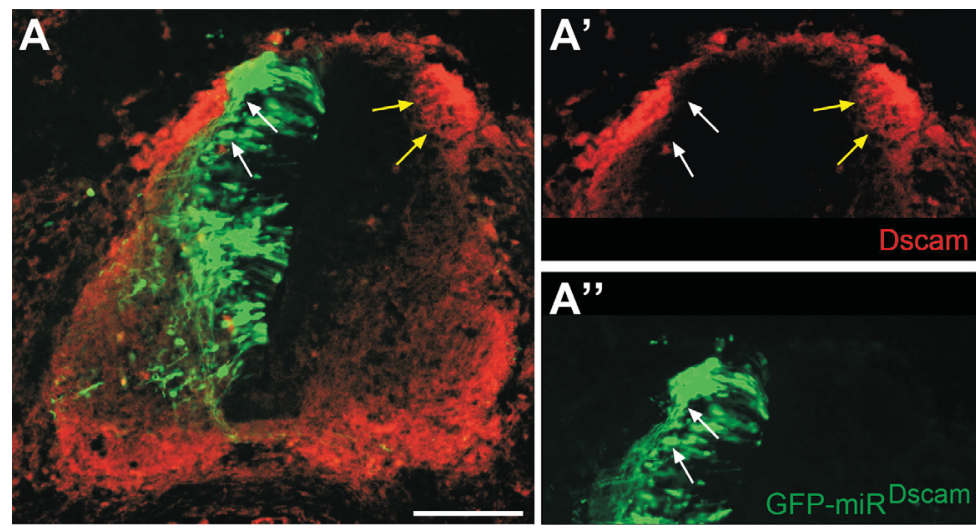

lateral and ventral dl2 neurons express Dscam (Fig. 1D). Isl1 was used as a dI3 neuronal marker. Consistently, expression of Dscam is restricted to the ventro/laterally migrating differentiated dI3 neurons (Fig. 1D). Hence, Dscam is expressed in the late differentiated dl1-3 neurons.

The dorsal interneurons vary in their axonal projection pattern. Along the dorsal/ventral axis, dl1c and dl2 project their axons contralaterally, while $\mathrm{dl} 1 \mathrm{i}$ and $\mathrm{dl} 3$ neurons project ipsilaterally (Fig. 1G). The expression of Dscam protein along the axons was studied utilizing Dscam-specific antibody (Yamagata and Sanes, 2008). Dscam protein is present in the dorsal root entry zone, consistent with its expression in the DRG, in the white matter, at the lateral and ventral funiculi, where the longitudinal ipsilateral axons project, and at the floor plate, the crossing zone of the commissural axons (Fig. 1F). Thus, Dscam is expressed in both commissural and ipsilaterally projecting neurons and along their axons.

Fig. 2. The effect of miR ${ }^{\text {Dscam }}$ on Down Syndrome cell adhesion molecul (Dscam) expression. GFP-miR ${ }^{D s c a m}$ cassette was expressed in the chick hemitube. A cross section stained with anti-Dscam is shown. At the dorsal spinal cord, neurons expressing miR $R^{\text {Dscam }}$ do not express Dscam (white arrows), while neurons at the dorsal part of the un-electroporated side express Dscam lyellow arrows). Scale bar in (A), $100 \mu \mathrm{m}$.

\section{Strategies for loss and gain of function of Dscam in the chick spinal cord}

We previously used a plasmid-based electroporation apspinal cord, we first characterized its spatial and temporal expression. In situ mRNA hybridization, combined with antibody staining for cell fate markers, was performed on E4-E6 chick embryo cross sections. At thoracic level of E4, Dscam mRNA is expressed in motor neurons and in ventral interneurons (Fig. 1A). Expression in motor neurons is observed in the thoracic-level motor neurons at E5 (Fig. 1B) and E6 (data not shown). Dscam is not expressed at $\mathrm{E} 5$ in the lateral motor column (LMC) neurons (Fig. 1 C,D). At E6, LMCI neurons express low levels, while LMCm neurons express high levels of Dscam (Fig. 1E). Expression in the dorsal interneurons is apparent at E5 (Fig. 1 C,D). Notably, at E4 the dorsal commissural neurons, whose axons reach the floor plate at this developmental stage, do not express detectable levels of Dscam (Fig. 1A).

We used cell type specific markers to verify the profile of Dscam expression in dl1-3 neurons. In situ double fluorescence hybridization to Lhx9 and Dscam reveals that the most dorsal dl1 neurons, the early-differentiated dl1 neurons, do not express Dscam, while the lateral and more ventral differentiated dl1 neurons express Dscam (Fig. 1B). The expression of Dscam in dl2 neurons was studied using Lhx1/5 antibody. The early-differentiated, dorsally/ medially located dI2 neurons, do not express Dscam, while the more proach for knock-down and ectopic expression of Dscam in the chick retina (Yamagata and Sanes, 2008). Here, we used micro-RNA targeting Dscam ( $\mathrm{miR}^{\mathrm{Dscam}}$ ) and and a DscamcDNA, both under the regulation of the CAGG enhancer/promoter or interneuron-specific enhancers, for loss and gain of function (Table 1). To assess our ability to down-regulate Dscamexpression in the spinal cord, spinal cords of chick embryos were electroporated at Hamburger-Hamilton Stage 18. At E6, embryos were removed, fixed and analyzed for Dscam protein expression. Electroporation of spinal neurons with $\mathrm{miR}^{\mathrm{Dscam}}$ decreased the expression of the Dscam in the soma and axons (Fig. 2A).

\section{Dscam is not required or sufficient for the selection of axonal trajectories by dl1 neurons}

To manipulate specific interneurons, we subcloned the $\mathrm{miR}^{\mathrm{D}}$ scam cassette in a Cre-dependent plasmid or a Cre/Gal4 double conditional plasmid (Avraham et al., 2009), using the approach of Wilson and Stoeckli (2011). For dl1-specific perturbations, the Cre-conditional miR ${ }^{\mathrm{Dscam}}$ plasmid was electroporated along with a Edl1::Cre plasmid (Avraham et al., 2009) (Table 1, Fig. 3E). The size of the spinal cords of miR ${ }^{\text {Dscam }}$ and control GFP-electroporated embryos was comparable, indicating no general effect on develop-

TABLE 1

\section{PLASMIDS USED FOR KNOCK-DOWN AND ECTOPIC EXPRESSION OF DSCAM}

\begin{tabular}{|c|c|c|c|c|}
\hline Neuron & Enhancers & Target plasmids for loss of function & Target plasmids for gain of function & Fig. \\
\hline \multirow{4}{*}{ dl1 } & \multirow{3}{*}{ Ed11::Cre } & $\begin{array}{l}\text { CAG::GFP-miR Rscam } \\
\text { CAG::STOP }{ }^{\text {LoxP }} \text {-taumyc }\end{array}$ & & 2B,D \\
\hline & & & CAG::Dscam-IRES-GFP & $2 \mathrm{C}$ \\
\hline & & CAG::STOP ${ }^{\text {LoxP }}$-GFP-miR ${ }^{\text {Uscam }}$ & & $3 B, F$ \\
\hline & Edl1::Gal4 & & UAS::mCherry-2A-Dscam & $3 \mathrm{D}, \mathrm{G}$ \\
\hline dl1 & $\begin{array}{l}\text { Edl1::Cre } \\
\text { Edl1::Gal4 }\end{array}$ & UAS::taumyc ${ }^{\text {LoxP }}-$ GFP-miR $R^{\text {Dscam }}$ & & $6 \mathrm{~B}$ \\
\hline \multirow{2}{*}{$\mathrm{d} 12$} & \multirow{2}{*}{$\begin{array}{c}\text { Edl1/2::Cre } \\
\text { Edl2/V1::Gal4 }\end{array}$} & UAS::STOP $P^{\text {LoxP }}-$ GFP-miR ${ }^{\text {Uscam }}$ & & 4B,E \\
\hline & & & UAS::STOP ${ }^{\text {LoxP }}$ - mCherry-2A- Dscam & $4 C, E$ \\
\hline \multirow{2}{*}{ dl3 } & \multirow{2}{*}{$\begin{array}{l}215:: \text { Gal } 4 \\
242:: \text { Cre }\end{array}$} & UAS::STOP ${ }^{\text {LoxP }}-$ GFP-miR ${ }^{\text {Uscam }}$ & & $5 B, E$ \\
\hline & & & UAS::STOP ${ }^{\text {LoxP }}$ - mCherry-2A- Dscam & $5 C, E$ \\
\hline
\end{tabular}


ment (Fig. 3 A-D). The ratio of ipsilaterally projecting axons from the total longitudinal axons did not differ between miR ${ }^{\text {Dscam }}$ and control GFP-electroporated embryos (Fig. 3A,B,F, Fig. S1A, Table 2). Likewise, the ectopic expression of Dscam in dl1 neurons did not alter the ipsi/contralateral ratio of dl1 axons. The ratio of ipsi/ total-projecting dl1 axons was similar between the Dscam overexpressing dl1 neurons and control dl1 neurons (Fig. 3 C,D,G, Fig. $\mathrm{S1B}$, Table 2). Thus, neither down- nor up-regulation of Dscam in dl1 neurons significantly affects the ipsi- versus contralateral choice of dl1 neurons.
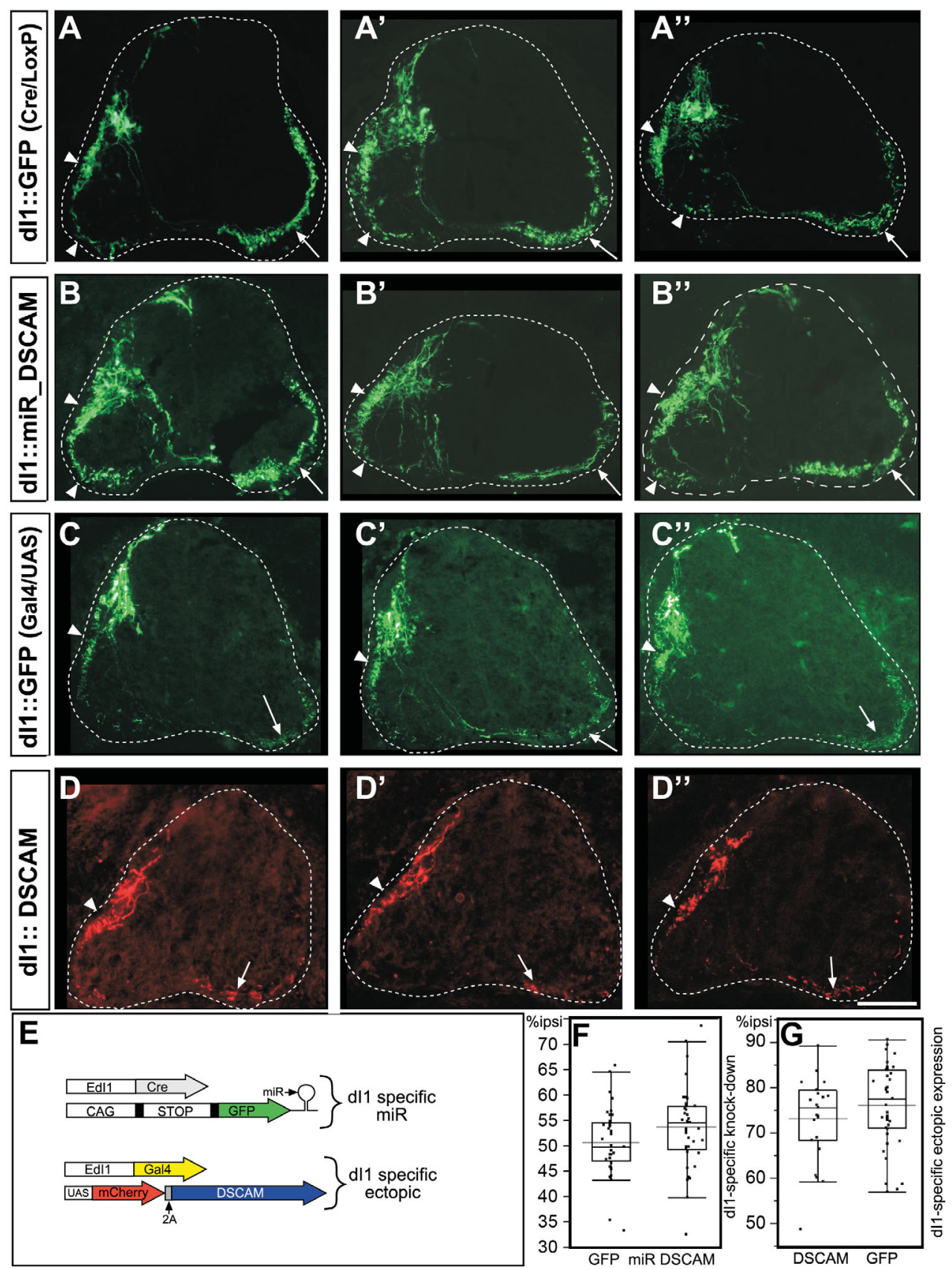

Fig. 3. Loss and gain of function of Dscam in dl1 neurons. Using the plasmids illustrated in (E), Dscam levels were down-(B) and up-(D) regulated. GFP expressed by the same drivers (Cre for $A, B$ and Gal4 for $C, D$ ) was used as a control. Three representative images from each group are shown. White arrows point to the contralaterally projecting axons. Arrowheads point to the ipsilaterally projecting axons. (E) Schemes showing the molecular strategies for manipulating Dscam levels in dl1 neurons. (F) Quantification of the ipsilaterally projecting dl1 axons following knockdown of Dscam in dl1 neurons. A t-test was performed to compare the adjusted \%ipsi of the miR Dscam to the Control group. The two groups do not appear to be significantly different for alpha=0.05 (p-value=0.074). (G) Quantification of the ipsilaterally projecting dl1 axons following ectopic expression of Dscam in dl1 neurons. A t-test was performed to compare the adjusted \%ipsi of the Dscam to the GFP group. The groups do not appear to be statistically different (p-value $=0.226)$. Box plot charts $(\mathbf{G}, \mathbf{H})$ : The boxes, enclose $50 \%$ of the results; the horizontal lines above and under the box enclose $90 \%$ of the results; the black and gray horizontal lines within the box are the average and the median, respectively. Scale bar in (D), $125 \mu \mathrm{m}$. 
TABLE 2

\section{SUMMARY OF THE STATISTICAL ANALYSIS}

\begin{tabular}{|c|c|c|c|c|c|c|c|}
\hline \multirow[b]{2}{*}{ Fig. } & \multirow[b]{2}{*}{ Statistical test } & \multirow[b]{2}{*}{ Dependent Variable } & \multirow[b]{2}{*}{ Significance } & \multicolumn{4}{|l|}{ Summary statistics ${ }^{\star}$} \\
\hline & & & & Group & $\mathbf{N}$ & Mean & Std Dev \\
\hline \multirow{2}{*}{$3 F$} & \multirow{2}{*}{ Dunnett's test } & \multirow{2}{*}{ \%ipsi } & \multirow{2}{*}{ No $(P=0.074)$} & GFP & 38 & 50.66 & 6.67 \\
\hline & & & & $\mathrm{miR}^{\text {Dscam }}$ & 39 & 53.70 & 7.94 \\
\hline \multirow[b]{2}{*}{$3 G$} & \multirow{2}{*}{ Dunnett's test } & \multirow{2}{*}{ \%ipsi } & \multirow{2}{*}{ No $(P=0.226)$} & DSCAM & 23 & 73.18 & 9.28 \\
\hline & & & & GFP & 41 & 76.07 & 8.99 \\
\hline \multirow{3}{*}{4} & \multirow{3}{*}{ Dunnett's test } & \multirow{3}{*}{$\%$ comm } & \multirow{3}{*}{$\begin{array}{l}\text { Yes, for both (multiple comparisons, controls for the } \\
\text { overall alpha level of } 0.05 \text {. see fig } 4 E \text { ) }\end{array}$} & GFP & 33 & 92.10 & 3.73 \\
\hline & & & & ectopic Dscam & 25 & 88.43 & 5.44 \\
\hline & & & & $\mathrm{miR}^{\text {Dscam }}$ & 53 & 89.30 & 5.85 \\
\hline \multirow{3}{*}{5} & \multirow{3}{*}{ Dunnett's test } & \multirow{3}{*}{ \%ipsi } & \multirow{3}{*}{$\begin{array}{l}\mathrm{miR}^{\mathrm{DSCAM}} \text { is different and ectopic DSCAMis not } \\
\text { significantly different from the control (multiple } \\
\text { comparisons, controls for the overall alpha level of } \\
0.05 \text {. see fig } 5 \mathrm{E} \text { ) }\end{array}$} & GFP & 21 & 97.44 & 0.99 \\
\hline & & & & ectopic Dscam & 16 & 97.13 & 1.52 \\
\hline & & & & $\mathrm{miR}^{\mathrm{Dscam}}$ & 35 & 98.19 & 0.84 \\
\hline 6 & paired T-Test & $\begin{array}{l}\text { The width of } \mathrm{miR}^{\mathrm{Uscam}} \text { and } \\
\text { taumyc axonal bundle }\end{array}$ & Yes $(P<0.0001)$ & $\mathrm{miR}^{\text {Dscam }}$ minus taumyc & 14 & 34.29 & 19.10 \\
\hline
\end{tabular}

*For Figures 3-5, after adjustment for embryo effect

\section{Dscam is not required or sufficient for the commissural axon projection of d12 neurons}

To direct dl2-specific knockdown in dl2 neurons, the miRDscam cassette was subcloned into a Gal4/Cre dual conditional vector. The dual conditional miR $^{\text {Dscam }}$ plasmid was co-electroporated with the dl2 enhancers plasmid-mix (Avraham et al., 2009) (Table 1, Fig. 4D). A robust commissural projection following Dscamknockdown was observed in dl2 neurons (Fig. 4A,B). The majority of dl2 axons projected contralaterally following dI2-specific expression of either GFP, miR ${ }^{\text {Dscam }}$, or Dscam (92.2+/-3.7\%, 89.3+/-5.9\% and $88.4 \%+/-5.4 \%$, respectively; Fig. 4 C,E, Fig. S2, Table 2). Statistical comparisons with a control using Dunnett's Method demonstrated a minor, though statistically significant, difference between the control and the manipulated dI2 axons. However, we observed no qualitative alteration in the trajectory of dl2 axons toward and across the floor plate. nor was there a significant difference in the commissural ratio between

Fig. 4. Loss and gain of function of Dscam in dl2 neurons. Using the plasmids illustrated in (D), Dscam levels were down- (B) and up- (C) regulated in $\mathrm{d} / 2$ neurons. GFP expressed by the same drivers was used as a contro/(A). Three representative images from each group are shown. White arrows point to the contralaterally projecting axons. (D) Schemes showing the molecular strategies for manipulating Dscam levels in dI2 neurons. (E) Quantification of the contralaterally projecting dI2 axons following knockdown and ectopic expression of Dscam. The difference between the ectopic and the miRDSCAM groups and the control group was assessed using the Dunnett's test (multiple comparisons). The test compares each level to the control level and controls for the overall alpha level (with an alpha of 0.05). The ectopic and the miR DSCAM groups are significantly different from the control group ( $p$-value $=0.017$ and 0.032 respectively). Box plot chart (E): The boxes, enclose $50 \%$ of the results; the horizontal lines above and under the box enclose $90 \%$ of the results; the black and gray horizontal lines within the box are the average and the median, respectively. Scale bar in (C), $125 \mu \mathrm{M}$.
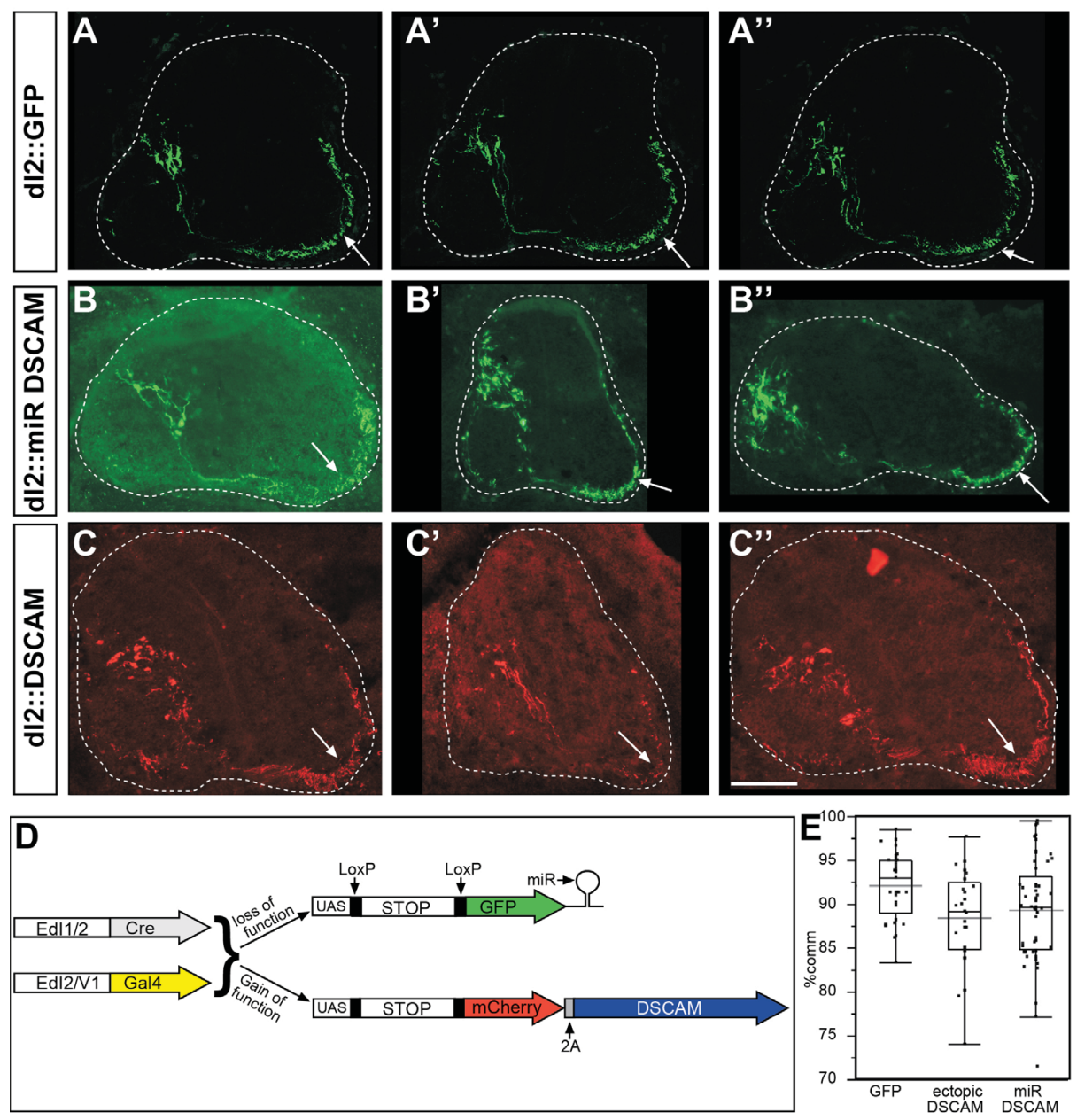
embryos, and $97.13+/-1.52 \%$ in the ectopic Dscamtreated embryos (Fig. 5E, Fig. S3, Table 2). Levels of crossing were significantly lower in the miRDscam group than in controls, but since $>97 \%$ of axons remained ipsilaterally in controls, the difference was of necessity small. Perhaps more noteworthy is the failure of Dscam overexpression to promote crossing of a statistically significant number of additional axons. Thus, our data provide little support for a role of Dscam in the crossing of dl3 axons.

\section{Dscam is required for axonal fasciculation}

The axons of dl1-3 neurons form cell type specific fascicles (Avraham et al., 2010a, Avraham et al., 2009). Dscam, which mediates homophilic interactions (Yamagata and Sanes, 2008), could contribute to fasciculation. To test Dscam's possible role in this process, we designed a double labeling paradigm that enables simultaneous detection of wild type and knock-down mutated neurons (WT/KD plasmid). A cassette of taumyc reporter gene flanked
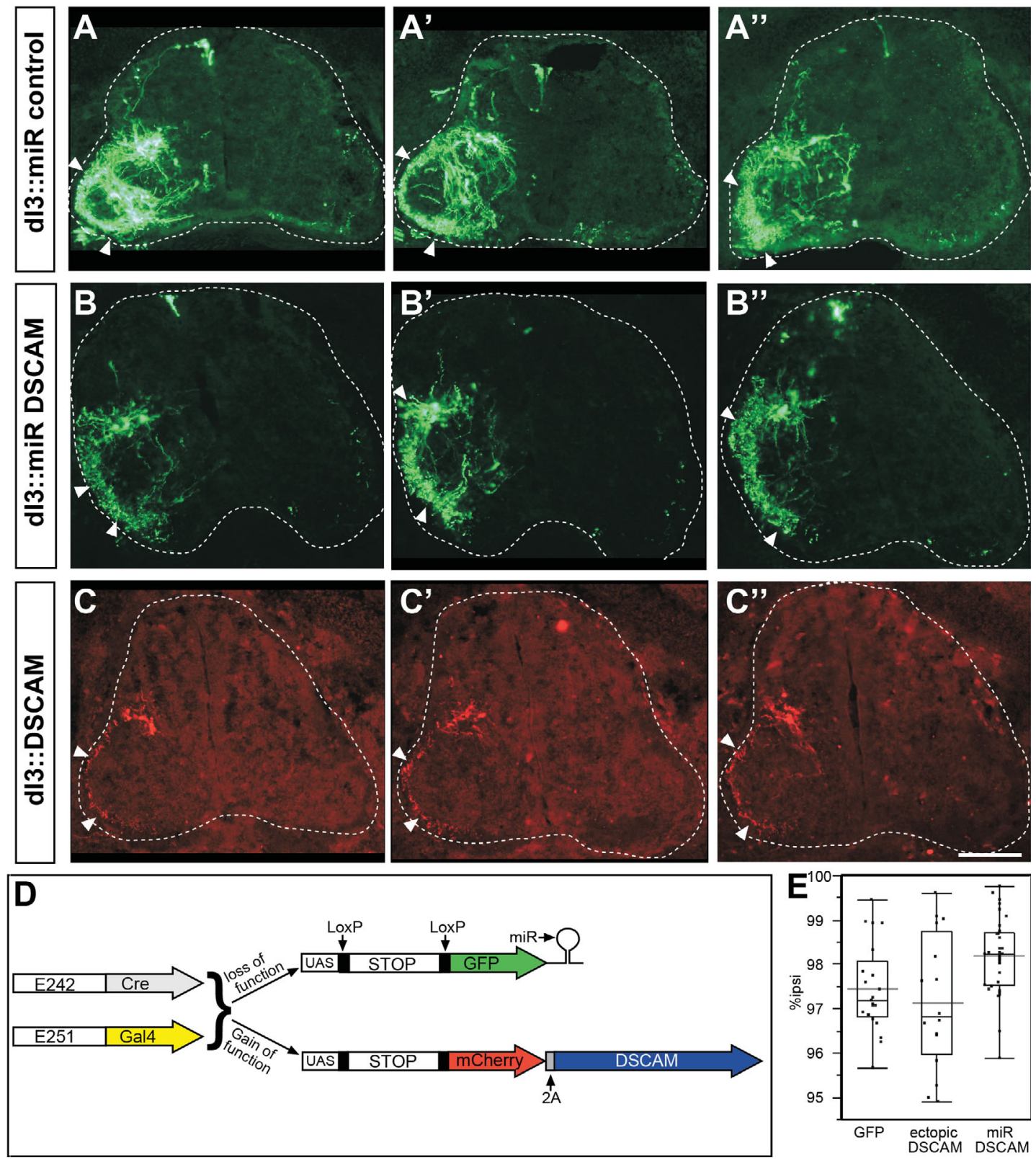

Fig. 5. Loss and gain of function of Dscam in dl3 neurons. Using the plasmids illustrated in (D), Dscam levels were down- (B) and up-(C) regulated in d/3 neurons. GFP expressed by the same drivers was used as a control (A). Three representative images from each group are shown. White arrowheads point to the ipsilaterally projecting axons. (D) Schemes showing the molecular strategies for manipulating Dscam levels in dI3 neurons. (E) Quantification of the ipsilaterally projecting dl3 axons following knockdown and ectopic expression of Dscam. The difference between the ectopic and the miR DSCAM groups and the control group was assessed using the Dunnett's test (multiple comparisons). The test compares each level to the control level and controls for the overall alpha level (with an alpha of 0.05). The miRDSCAM group is significantly different than the control (p-value=0.026) whereas the ectopic group is not statistically significantly different (p-value=0.581). Box plot chart (E): The boxes, enclose $50 \%$ of the results; the horizontal lines above and under the box enclose $90 \%$ of the results; the black and gray horizontal lines within the box are the average and the median, respectively. Scale bar in (C), $125 \mu \mathrm{M}$. 
by LoxP sites and proceeded by EGFP (EGFP-miR ${ }^{\text {Dscam }}$ ) was cloned downstream to UAS promoter (Fig. 6C). The WT/KD plasmid was co-electroporated with Ed11-driven Cre and Gal4 plasmids to drive expression in dl1 neurons. Neurons that incorporate all the three plasmids will express EGFP-miR ${ }^{\text {Dscam }}$ and consequently will down regulate Dscam expression, while neurons that incorporate only the Edl1::Gal4 and WT/KD plasmids will express taumyc. Other combination of plasmids will not lead to expression of either the reporter or the miR ${ }^{\text {Dscam }}$ cassette. We excluded axons that expressed both EGFP and taumyc; they likely initiated expression of taumyc early, and only subsequently activated the miR ${ }^{\text {Dscam }}$ via late expression of Cre.

The main result was that the Dscam-deficient (EGFP-positive) axons were spread over a broader area than the control (taumycpositive) axons (Fig. 6A,B). To quantify the difference, we measured the width (in pixels) of the dl1 axon bundle at the circumferential axis (Fig. 6D). The average width of miRDscam dl1 bundle was $2.05+/-0.86$ times greater than the width of the control bundle.

Due to the fact that the wild type and mutated axons differ from each other regarding their fascicule width, the statistical analysis was preformed in the following manner: the width of miR ${ }^{\text {Dscam }}$ and the control (taumyc expressing axons) were scored per section.
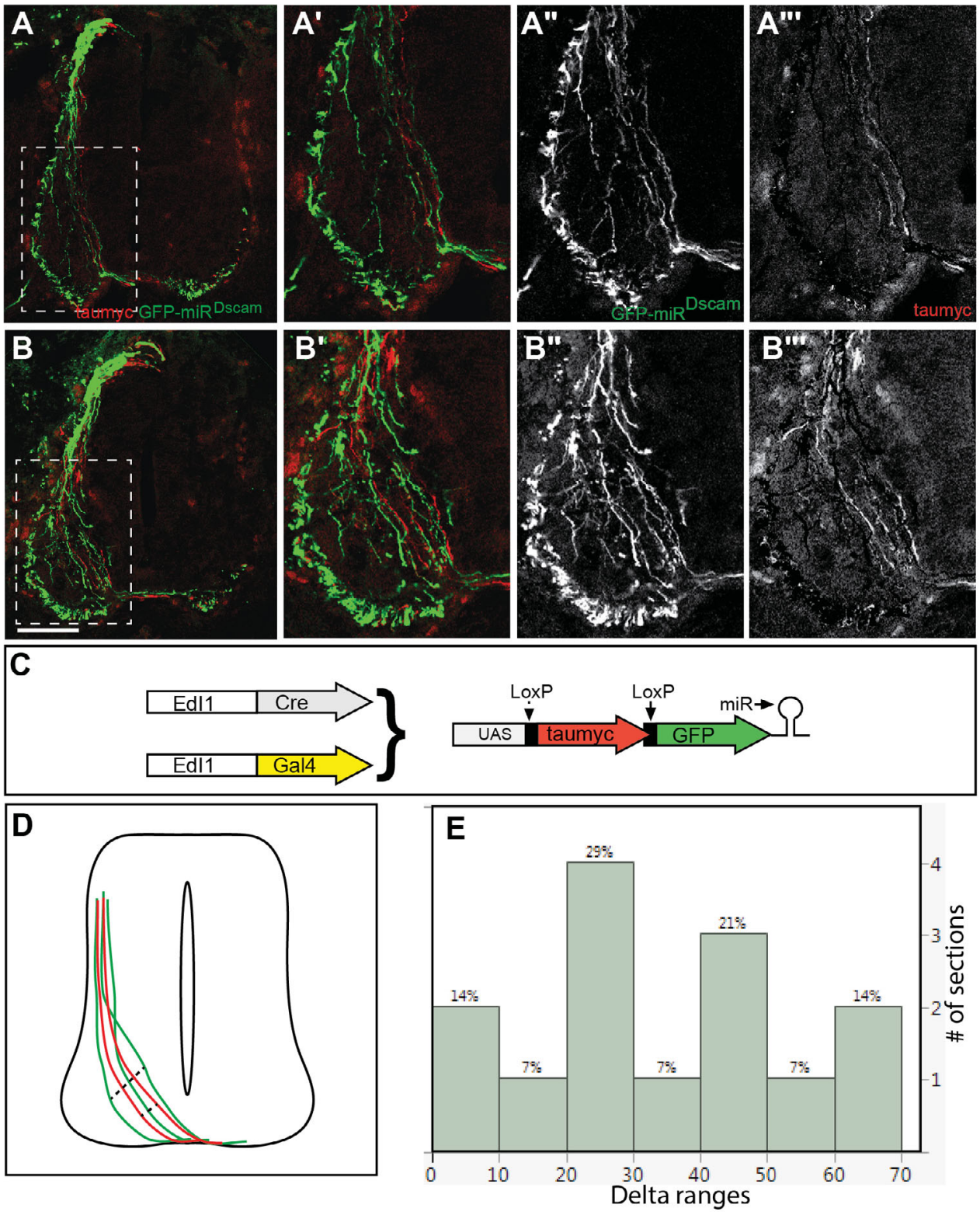

Fig. 6. Knockdown of Dscam results in widening of dl1 axonal fascicle. Using the plasmids illustrated in (C), a chimeric axonal genotype of dl1 neurons was obtained: Wild type dl1 neurons expressing miR ${ }^{D S C A M}$ and mutated dl1 neurons expressing GFP-miR ${ }^{D S C A M}$. The wide fanning of GFP-miR ${ }^{D S C A M}$ axons (A,A','A",B, $\left.\mathbf{B}^{\prime} \mathbf{B}^{\prime \prime}\right)$ versus the tight bundle of the wild type, taumyc-expressing axons ( $\left.\mathbf{A}^{\prime \prime \prime}, \mathbf{B}^{\prime \prime \prime}\right)$, is evident. The width of the bundles was measured as illustrated (D). The chart (E) shows the histogram of the differences. The $X$ axis shows delta ranges - the width of the miRDscam fascicule minus the width of taumyc fascicule (width measured as pixels). The $Y$ axis shows the the counts (number of samples). Above each bar the percent of sections is specified. Scale bar in (A), $100 \mu \mathrm{m}$ for (A) and $60 \mu \mathrm{m}$ for $\left(A^{\prime}-A^{\prime \prime \prime}\right)$. 
Deltas (the width of $\mathrm{miR}^{\mathrm{Dscam}}$ fascicle minus taumyc fascicle) were evaluated per image. Thus, if the two groups widths were similar, these deltas would tend to be distributed around zero, while larger deltas would indicate larger $\mathrm{miR}^{\mathrm{Dscam}}$ widths compared to the control. Deltas evaluated from the 14 sections, range between 7 and 32 with an average of $34.3+/-19.1$. A T-Test was used for testing the null hypothesis that the deltas are zero. The test showed a statistically significant result, rejecting the null hypothesis $(P<0.0001)$. (Fig. 6E, table 2$)$. Therefore, we concluded that the width of $\mathrm{miR}^{\mathrm{Dscam}} \mathrm{dl} 1$ axons was significantly larger than that of control axons. Thus, Dscam is required for the fasciculation of dl1 axons as they project toward the floor plate.

\section{Discussion}

Genetic evidence in the fly supports the role of Dscam in the guidance of embryonic commissural axons by Netrin-1 dependent and independent modes (Andrews et al., 2008). In vertebrates, down-regulation of Dscam, via siRNA treatment, was shown to inhibit the axonal growth of dorsal interneurons toward the floor plate in mouse and chick (Liu et al., 2009, Ly et al., 2008). Conversely, the in vivo requirement of Dscam for commissural guidance in vertebrates was contested in a recent paper that used a null allele, which reported a normal projection pattern of spinal commissural axons in the (Palmesino et al., 2012).

We have reexamined the role of Dscam in the guidance of chick spinal interneurons using cell-type specific knock-down and ectopic expression strategies. The loss and gain of function tools used in this study were previously utilized to demonstrate the requirement of Dscam for directing the lamina-specific synaptic connection of Dscam-expressing retinal ganglion cells (Yamagata and Sanes, 2008). A Knock-down of Dscam in the commissural interneurons, dl1 $\mathrm{c}$ and $\mathrm{dl} 2$, did not prevent their extension toward and across the floor plate. Complementary, ectopic expression of Dscam in the ipsilaterally projecting populations, dl1i and dl3, did not alter their ipsilateral projection. Though, we argue that as in the mouse, also in the chick spinal cord Dscam is not involved in attraction to the floor plate. In addition, we have demonstrated that the ipsilateral projection of $\mathrm{dl} 1 \mathrm{c}$ and $\mathrm{dl} 3$ neurons is not altered following Dscam knockdown, and the commissural projection of dl1c and dl2 is not affected succeeding ectopic expression of Dscam. Providing evidence for the dispensability of Dscam for midline repulsion of ipsilateral projecting neurons, which was not addressed in the mouse genetic model (Palmesino et al., 2012). Our results do not provide substantial support for the idea that Dscam serves as a neuronal receptor for cues that promote midline crossing of spinal interneurons. In this respect, our conclusions are consistent with those of Palmesino et al., (2008). On the other hand, we did observe small but statistically significant decreases or increase in crossing when Dscam levels were decreased in dl2 and dl3 neurons, respectively. We were unable to obtain a quantitative measure of the extent of Dscam attenuation in these neurons. Therefore, we cannot formally exclude the possibility that more complete inhibition might have led to a more dramatic effect. New gene editing tools will provide a means of testing this possibility in the future.

The binding of netrin-1 to Dscam was shown using diverse biochemical and cell biology means (Andrews et al., 2008, Liu et al., 2009, Ly et al., 2008). Genetic evidence in flies supports the role of Dscam in mediating attraction toward the midline-derived Netrin molecules (Andrews et al., 2008). An additional interpretation for Netrin/Dscam interaction is suggested: Dscam1 functions to counter Drosophila sensory neuron dendritic targeting signals provided by secreted Netrin-B and Frazzled. Loss of Dscam 1 function resulted in Frazzled-dependent aberrant dendrite accumulation at a Netrin-B-expressing target (Matthews and Grueber, 2011). The counter-netrin activity of Dscam may reside from competition on Netrin binding between Dscam and Fra/DCC. Since the affinity to Netrin of both receptors is similar, higher levels of Dscam will sequester Netrin from binding the Fra/DCC. Alternatively, Dscammediated self-avoidance that is triggered by homophilic binding of Dscam, may initiate a signaling cascade that inhibits the Netrin/ DCC signaling pathway. The relative balance between the levels of Dscam and DCC can shift the equilibrium between attraction and repulsion in this model as well.

Netrin-1/Dscam interaction in vertebrates may modulate Netrin-1 activity at other, post floor plate, choice points along the pathway. The possible role of Dscam in the guidance of the axons along the late longitudinal segments rather than the early circumferential segment of their projection, relies on the temporal profile of Dscam expression. At E4, when commissural axons cross the floor plate, Dscam is not expressed in the dorsal interneurons (Fig. 1A). At E5, Dscam expression is absent in the early-differentiated commissural interneurons that project toward the floor plate, while its expression is up-regulated in the presumed post-crossing late-differentiated neurons (Fig. 1 B-D). Dscam may participate in silencing netrin-1 responsiveness in post-floor plate-crossing commissural axons.

Homophilic interaction between Dscam expressing neurons was demonstrated in the chick and mouse retina. Our results highlight the homophilic activity also in the wiring of spinal interneurons. The novel wild type/knockdown chimeric system (Fig. 6) that we have developed assisted in revealing the requirement of Dscam for axonal fasciculation of dl1 axons. Scoring the phenotype of wild type and mutated neurons, within the same specimen, is advantageous over using control and manipulated chick embryos, since the efficiency of gene delivery may vary significantly between embryos. Applying this method is currently limited to neurons that can be targeted by a single enhancer. Hence, the possible role of Dscam in the fasciculation of $\mathrm{dl} 2$ and $\mathrm{dl} 3$, as well as in other spinal interneurons was not addressed in the current study. Therefore, we cannot exclude a dl1-specific role of Dscam in axonal fasciculation. Notably, no fasciculation defects, in TAG expressing commissural axons, were reported in the Dscam null mouse (Palmesino et al., 2012). The discrepancy may arise from the fact that dl1 are only a fraction of commissural axons, and their projection is obscure by other commissural axons.

The responsiveness of growth cones to guidance cues is context-dependent. Repulsion from-versus attraction to- a defined guidance molecule can be gated by different receptor complexes or different intracellular signaling pathways (Dickson and Zou, 2010, Evans and Bashaw, 2010). Similarly, adhesive and repellent interactions between axons, that leads to either fasciculation or defasciculation, respectively, are also context-dependent. Role for Dscam in adhesive interaction in the retina (Yamagata and Sanes, 2008), as well as a repulsive molecule that prevents fasciculation of RGC's dendrites (Fuerst et al., 2009), was demonstrared. Likewise, in Drosophila, Dscam is implicated in dendrites avoidance 
(Matthews et al., 2007) as well as attraction (Tadros et al., 2016). It is not yet clear what controls the opposing activities of Dscam.

Dscam is expressed in numerous interneurons in the spinal cord, which raises the question whether Dscam can serve as a cell type specific fasciculation factor. Expression of other lg-super family molecules that share the homophilic interaction activity: Sidekicks and Contactins, is more restricted in the spinal cord. Hence, a combinatorial expression of DSCAM/Sdk and Contactins may serve as homophilic code for self fasciculation of axonal bundle in the spinal cord, as it was shown in the retina (Yamagata and Sanes, 2012).

\section{Materials and Methods}

\section{DNA}

The GFP-miR-Dscam-b cassette (Yamagata and Sanes, 2008), was amplified by PCR using forward [ATGCTAGCAAAACCATGGTGAGCAAGGG] and reverse [ATGCGGCCGCTAGATATCTCGAGTGCGGCC ] primers. PCR product was digested with Nhel and Notl and subcloned into a Cre or Gal4/Cre dependent plasmids (Avraham et al., 2009, Hadas et al., 2014). The mCherry-2A-Dscam cassette (Yamagata and Sanes, 2008) was excised by EcoRI+Notl digest and subcloned downstream to the UAS promoter. Plasmids containing dl1, dl2 and dl3 enhancers driving Cre and Gal4 were described previously (Avraham et al., 2010a, Avraham et al., 2009, Hadas et al., 2014).

\section{In ovo electroporations}

Fertilized White Leghorn chicken eggs were incubated at $38.5-39^{\circ} \mathrm{C}$. A DNA solution of $5 \mathrm{mg} / \mathrm{ml}$ was injected into the lumen of the neural tube stage 17-18. Electroporation was performed using $3 \times 50 \mathrm{~ms}$ pulses at $30 \mathrm{~V}$, applied across the embryo using a $0.5 \mu \mathrm{m}$ Tungsten wire and a BTX electroporator (ECM 830). Embryos were incubated for 3 days prior to analysis (Avraham et al., 2010b).

\section{Immunohistochemistry}

Embryos were fixed overnight at $4^{\circ} \mathrm{C}$ in $4 \%$ paraformaldehyde/ $0.1 \mathrm{M}$ phosphate buffer, washed twice with PBS, incubated in $30 \%$ sucrose/ PBS for 24 hours, and embedded in OCT. Cryostat sections (14 $\mu \mathrm{m})$ were collected on Superfrost Plus slides and kept at $-70^{\circ} \mathrm{C}$. The following antibodies were used: rabbit polyclonal GFP antibody (Molecular Probes), Lhx2/9, Lhx1/5, Isl1 (provided by T. Jessell, Columbia University, New York, NY) Dscam (Medimabs, Montreal). Cy2, RRX and cy5 were used as fluorochromes. Images were captured under a microscope (Axioscope 2; Zeiss) with a digital camera (DP70; Olympus) or confocal microscope (FV1000; Olympus).

For in situ double mRNA fluorescence the Tyramide Signal Amplification (TSA) kit (Perkin Elmer) was used. For double: mRNA in situ + immunohistochemistry, the traditional dig-AP (Roche) protocol was used. Following the AP color reaction, slides were incubated with the appropriate antibody as described above.

\section{Quantification}

Each experiment included both manipulated and control groups, which were electroporated within two hours of each other. Comparisons were made between manipulated and control embryos within an experiment. For each group 2-5 embryos were electroporated. At E6 embryos were removed from the eggs, fixed and sectioned. In each cross section the number of axons projecting longitudinally was scored. The number of pixels in the white matter at the ipsilateral and contralateral was calculated using ImageJ tools.

Analysis methodology: In some instances, there was a large embryo to embryo variability within groups. Thus, the analyses were performed on adjusted data, after removing the embryo effect. T-test was applied on the adjusted values to compare between two groups. In the cases of three groups, where each level is compared to a control, the multiple comparisons Dunnet's test was used. The charts show the adjusted values. In the supplementary figures, both the original values and the embryo data are presented.

\section{Acknowledgements}

The authors thank Artur Kania, Oshri Avraham and Yoav Hadas for comments on the manuscript. This work was supported by grants to AK and JS from the BSF (2013/058), AK from the Israel Science Foundation (grant No. 229/09 and 631/13) and AK from the Ida and Avraham Baruch Endowment Fund.

\section{References}

ANDREWS, G.L., TANGLAO, S., FARMER, W.T., MORIN, S., BROTMAN, S. BERBEROGLU, M.A., PRICE, H., FERNANDEZ, G.C., MASTICK, G.S., CHARRON, F. et al., (2008). Dscam guides embryonic axons by Netrin-dependent and -independent functions. Development 135: 3839-3848.

AVRAHAM, O., HADAS, Y., VALD, L., HONG, S., SONG, M.R. and KLAR, A. (2010a). Motor and dorsal root ganglia axons serve as choice-points for the ipsi-lateral turning of dl3 axons. J. Neurosci. 30: 15546-15557.

AVRAHAM, O., HADAS, Y., VALD, L., ZISMAN, S., SCHEJTER, A., VISEL, A. and KLAR, A. (2009). Transcriptional control of axonal guidance and sorting in dorsal interneurons by the Lim-HD proteins Lhx9 and Lhx1. Neural Dev 4: 21.

AVRAHAM, O., S. ZISMAN, Y. HADAS, L. VALD and A. KLAR (2010). Deciphering axonal pathways of genetically defined groups of neurons in the chick neural tube utilizing in ovo electroporation. J Vis Exp 39: 1792. (doi: 10.3791/1792)

CHARRON, F., STEIN, E., JEONG, J., MCMAHON, A.P. and TESSIER-LAVIGNE, M. (2003). The morphogen sonic hedgehog is an axonal chemoattractant that collaborates with netrin-1 in midline axon guidance. Cell 113: 11-23.

DICKSON, B.J. and ZOU, Y. (2010). Navigating intermediate targets: the nervous system midline. Cold Spring Harb Perspect Biol 2: a002055.

EVANS, T.A. and BASHAW, G.J. (2010). Axon guidance at the midline: of mice and flies. Curr Opin Neurobiol 20: 79-85

FAZELI, A., DICKINSON, S.L., HERMISTON, M.L., TIGHE, R.V., STEEN, R.G. SMALL, C.G., STOECKLI, E.T., KEINO-MASU, K., MASU, M., RAYBURN, H. et al., (1997). Phenotype of mice lacking functional Deleted in colorectal cancer (Dcc) gene. Nature 386: 796-804.

FUERST, P.G., BRUCE, F., TIAN, M., WEI, W., ELSTROTT, J., FELLER, M.B., ERSKINE, L., SINGER, J.H. and BURGESS, R.W. (2009). DSCAM and DSCAML1 function in self-avoidance in multiple cell types in the developing mouse retina. Neuron 64: 484-497.

GOWAN, K., HELMS, A.W., HUNSAKER, T.L., COLLISSON, T., EBERT, P.J., ODOM R. and JOHNSON, J.E. (2001). Crossinhibitory activities of Ngn1 and Math1 allow specification of distinct dorsal interneurons. Neuron 31: 219-232.

HADAS, Y., ETLIN, A., FALK, H., AVRAHAM, O., KOBILER, O., PANET, A., LEVTOV, A. and KLAR, A. (2014). A 'tool box' for deciphering neuronal circuits in the developing chick spinal cord. Nucleic Acids Res. 42: e148.

HELMS, A.W. and JOHNSON, J.E. (1998). Progenitors of dorsal commissural interneurons are defined by MATH1 expression. Development 125: 919-928.

KENNEDY, T.E., SERAFINI, T., DE LA TORRE, J.R. and TESSIER-LAVIGNE, M. (1994). Netrins are diffusible chemotropic factors for commissural axons in the embryonic spinal cord. Cell 78: 425-435.

LEMIEUX, M., O, D.L., THIRY, L., BOULANGER-PIETTE, A., FRENETTE, J. and BRETZNER, F. (2016). Motor hypertonia and lack of locomotor coordination in mutant mice lacking DSCAM. J Neurophysiol 115: 1355-1371.

LIU, G., LI, W., WANG, L., KAR, A., GUAN, K.L., RAO, Y. and WU, J.Y. (2009). DSCAM functions as a netrin receptor in commissural axon pathfinding. Proc Natl Acad Sci USA 106: 2951-2956.

LY, A., NIKOLAEV, A., SURESH, G., ZHENG, Y., TESSIER-LAVIGNE, M. and STEIN, E. (2008). DSCAM is a netrin receptor that collaborates with DCC in mediating turning responses to netrin-1. Cell 133: 1241-1254.

MATTHEWS, B.J. and GRUEBER, W.B. (2011). Dscam1-Mediated Self-Avoidance Counters Netrin-Dependent Targeting of Dendrites in Drosophila. Curr Biol 21: 1480-1487. 
MATTHEWS, B.J., KIM, M.E., FLANAGAN, J.J., HATTORI, D., CLEMENS, J.C., ZIPURSKY, S.L. and GRUEBER, W.B. (2007). Dendrite self-avoidance is controlled by Dscam. Cell 129: 593-604.

OKADA, A., CHARRON, F., MORIN, S., SHIN, D.S., WONG, K., FABRE, P.J., TESSIER-LAVIGNE, M. and MCCONNELL, S.K. (2006). Boc is a receptor for sonic hedgehog in the guidance of commissural axons. Nature 444: 369-373.

PALMESINO, E., HADDICK, P.C., TESSIER-LAVIGNE, M. and KANIA, A. (2012). Genetic analysis of DSCAM's role as a Netrin-1 receptor in vertebrates. $J$ Neurosci 32: 411-416.

REEBER, S.L., SAKAI, N., NAKADA, Y., DUMAS, J., DOBRENIS, K., JOHNSON, J.E. and KAPRIELIAN, Z. (2008). Manipulating Robo expression in vivo perturbs commissural axon pathfinding in the chick spinal cord. J Neurosci 28: 8698-8708.

RUIZ DE ALMODOVAR, C., FABRE, P.J., KNEVELS, E., COULON, C., SEGURA, I., HADDICK, P.C., AERTS, L., DELATTIN, N., STRASSER, G., OH, W.J. et al., (2011). VEGF mediates commissural axon chemoattraction through its receptor Flk1. Neuron 70: 966-978.

SERAFINI, T., COLAMARINO, S.A., LEONARDO, E.D., WANG, H., BEDDINGTON, R., SKARNES, W.C. and TESSIER LAVIGNE, M. (1996). Netrin-1 is required for commissural axon guidance in the developing vertebrate nervous system. Cell 87: 1001-1014.
SERAFINI, T., KENNEDY, T.E., GALKO, M., MIRZAYAN, C., JESSELL, T.M. and TESSIER-LAVIGNE, M. (1994). The netrins define a family of axon outgrowthpromoting proteins homologous to C. elegans UNC-6. Cell 78: 409-424.

TADROS, W., XU, S., AKIN, O., YI, C.H., SHIN, G.J., MILLARD, S.S. and ZIPURSKY, S.L. (2016). Dscam Proteins Direct Dendritic Targeting through Adhesion. Neuron 89: 480-493.

THIRY, L., LEMIEUX, M., O, D.L. and BRETZNER, F. (2016). Role of DSCAM in the development of the spinal locomotor and sensorimotor circuits. I Neurophysiol 115: 1338-1354.

WILSON, N.H. and STOECKLI, E.T. (2011). Cell type specific, traceable gene silencing for functional gene analysis during vertebrate neural development. Nucleic Acids Res.

WILSON, S.I., SHAFER, B., LEE, K.J. and DODD, J. (2008). A molecular program for contralateral trajectory: Rig-1 control by LIM homeodomain transcription factors. Neuron 59: 413-424.

YAMAGATA, M. and SANES, J.R. (2008). Dscam and Sidekick proteins direct lamina-specific synaptic connections in vertebrate retina. Nature 451: 465-469.

YAMAGATA, M. and SANES, J.R. (2012). Expanding the Ig superfamily code for laminar specificity in retina: expression and role of contactins. J Neurosci 32 : 14402-14414. 


\section{Further Related Reading, published previously in the Int. J. Dev. Biol.}

The involvement of three signal transduction pathways in botryllid ascidian astogeny, as revealed by expression patterns of representative genes

Amalia Rosner, Gilad Alfassi, Elizabeth Moiseeva, Guy Paz, Claudette Rabinowitz, Ziva Lapidot, Jacob Douek, Abraham Haim and Baruch Rinkevich

Int. J. Dev. Biol. (2014) 58: 677-692

From Agrobacterium to viral vectors: genome modification of plant cells by rare cutting restriction enzymes Ira Marton, Arik Honig, Ayelet Omid, Noam De Costa, Elena Marhevka, Barry Cohen, Amir Zuker and Alexander Vainstein Int. J. Dev. Biol. (2013) 57: 639-650

What Hydra can teach us about chemical ecology - how a simple, soft organism survives in a hostile aqueous environment Tamar Rachamim and Daniel Sher Int. J. Dev. Biol. (2012) 56: 605-611

Analysis of chemotaxis when the fraction of responsive cells is small - application to mammalian sperm guidance Anna Gakamsky, Edna Schechtman, S. Roy Caplan and Michael Eisenbach

Int. J. Dev. Biol. (2008) 52: 481-487

Mouse models to study inner ear development and hereditary hearing loss Lilach M. Friedman, Amiel A. Dror and Karen B. Avraham Int. J. Dev. Biol. (2007) 51: 609-631

The importance of the posterior midline region for axis initiation at early stages of the avian embryo

Oded Khaner

Int. J. Dev. Biol. (2007) 51: 131-137

Early stages of neural crest ontogeny: formation and regulation of cell delamination Chaya Kalcheim and Tal Burstyn-Cohen

Int. J. Dev. Biol. (2005) 49: 105-116

Allogeneic interactions in Hydractinia: is the transitory chimera beneficial? Sharon Gild, Uri Frank and Ofer Mokady

Int. J. Dev. Biol. (2003) 47: 433-438

High proliferation rate characterizes the site of axis formation in the avian blastula-stage embryo.

$\mathrm{N}$ Zahavi, V Reich and O Khaner

Int. J. Dev. Biol. (1998) 42: 95-98
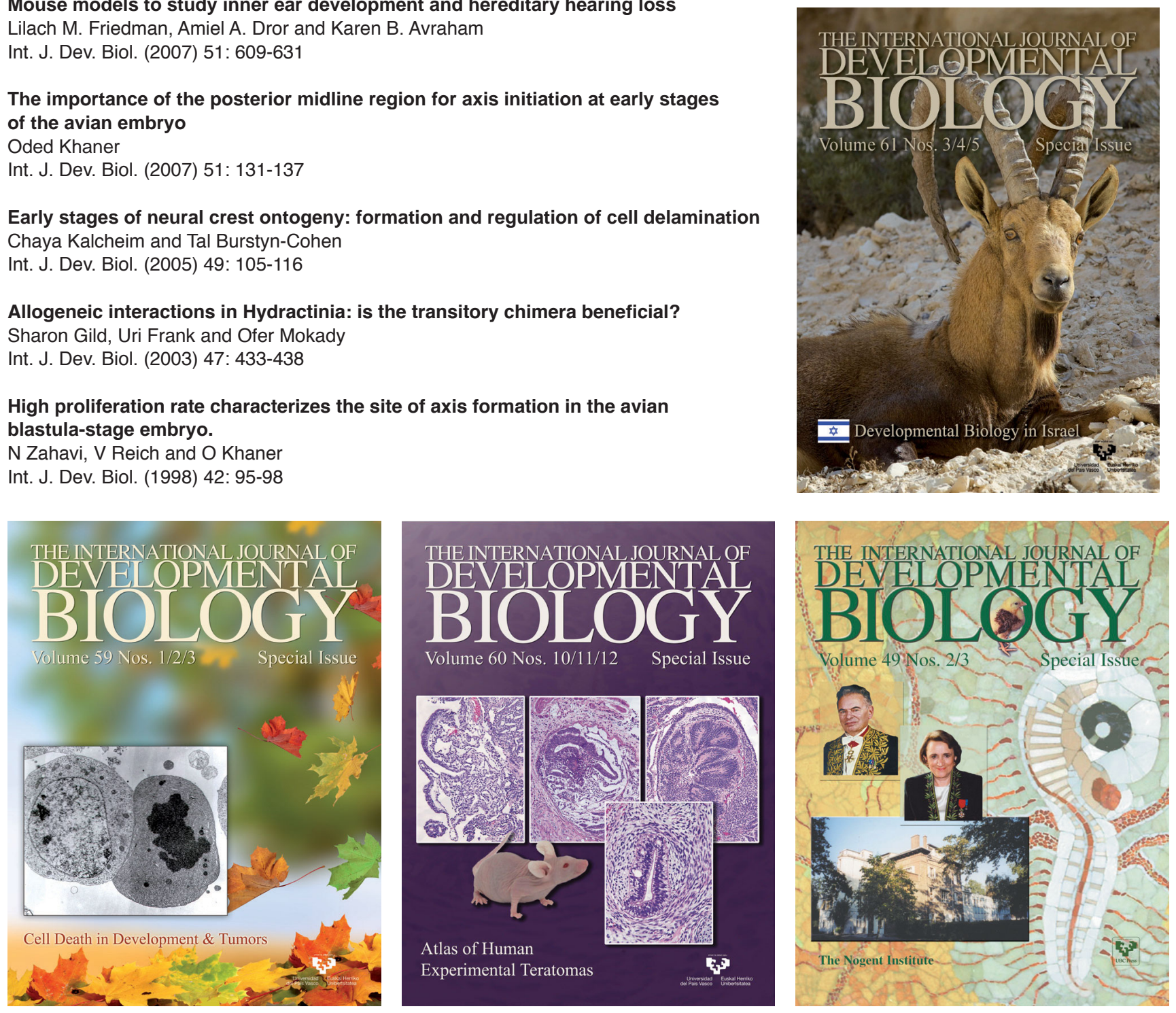